\title{
Variações fenotípicas em Andira fraxinifolia Benth. (Fabaceae) em duas fitofisionomias de Restinga
}

\author{
Karolline Raimundo da Silva ${ }^{1}$, João Carlos Ferreira de Melo Júnior ${ }^{1,3}$ e Maria Regina Torres Boeger ${ }^{2}$
}

Recebido: 25.11.2015; aceito: 14.04.2016

\begin{abstract}
Phenotypic variations of Andira fraxinifolia Benth. (Fabaceae) from two Restinga sandbank formations). The study analyzed the variability of morphological trait variability of Andira fraxinifolia from two different Restinga formations. The study was conducted at Acaraí State Park, São Francisco do Sul, Santa Catarina, Brazil, in areas of shrubtree Restinga and Forest transition. Five individuals were selected on each area in order to characterize their height, trunk diameter at breast height, leaf morphological traits, and stem anatomical traits. Andira fraxinifolia presented phenotypic variations between the studied areas; leaf dimensions and the plant size being the most significant variations. The structural adjustments of these traits can be adaptive, allowing the plant survival in areas with distinct environmental conditions. Keywords: adaptive adjustments, "angelim", ecotypes, heterogeneous environments
\end{abstract}

RESUMO - (Variações fenotípicas em Andira fraxinifolia Benth. (Fabaceae) em duas fitofisionomias de Restinga). O estudo analisou a variabilidade de caracteres morfológicos de Andira fraxinifolia (Fabaceae) em duas formações de Restinga. Os espécimes foram coletados no Parque Estadual do Acaraí, São Francisco do Sul, SC, Brasil, em áreas de vegetação de Restinga arbustivo-arbórea e de floresta de transição. Cinco indivíduos foram selecionados em cada área para caracterização da altura da planta, do diâmetro à altura do peito, da morfologia das folhas e da anatomia do lenho caulinar. Andira fraxinifolia apresentou variações fenotípicas significativas entre as áreas estudadas, com destaque às dimensões das folhas e o tamanho da planta. Estes ajustes estruturais podem ser adaptativos, promovendo a sobrevivência da espécie em locais com condições ambientais distintas.

Palavras chave: ajustes adaptativos, angelim, ecótipos, ambientes heterogêneos

\section{Introdução}

A Restinga, ecossistema típico da planície costeira brasileira, ocupa aproximadamente $70 \%$ do litoral do país e sua vegetação compreende um conjunto de comunidades vegetais fisionomicamente distintas, desde vegetação herbácea até formações florestais, definidas, em geral, pelo distanciamento da influência marinha (Araújo 1992). Plantas que habitam a Restinga estão sob condições ambientais mais extremas do que aquelas presentes em florestas mésicas, tais como grande amplitude térmica (incluindo o congelamento), oscilações entre períodos de inundação e seca, ventos constantes, alta salinidade e restrição de nutrientes (Scarano et al. 2001, Scarano 2002). Estas características ambientais podem induzir diferentes padrões de variações fenotípicas nas plantas que, por sua vez, permitem sua adequação ao meio físico e sobreviver em condições adversas (Schlichting 1986).

Variações fenotípicas mais notórias são observadas na folha, órgão mais exposto no vegetal e tido como um relevante indicador ecológico em função da expressão de fenótipos tão variados quanto as condições abióticas às quais está submetido (Peppe et al. 2011). Portanto, as folhas podem ser consideradas um órgão altamente plástico e aclimatável aos fatores ambientais (Gratani et al. 2006), sendo a intensidade luminosa, a disponibilidade hídrica e a temperatura os principais fatores que podem influenciar suas variações (Esau 1977). O caule também tem a capacidade de registrar na madeira os eventos microclimáticos e variações

1. Universidade da Região de Joinville, Laboratório de Anatomia e Ecologia Vegetal, Departamento de Ciências Biológicas, Rua Paulo Malschitzki, 10, Zona Industrial Norte, 89219-710 Joinville, SC, Brasil

2. Universidade Federal do Paraná, Laboratório de Ecologia e Morfologia Funcional, Setor de Ciências Biológicas, Caixa Postal 19031, 81531-990 Curitiba, PR, Brasil

3. Autor para correspondência: jcmelo_wood@hotmail.com 
sazonais no ambiente, como a oferta ou a escassez de nutrientes minerais e água (Kedrov 2012).

Nessa perspectiva, alguns estudos vêm descrevendo características de distintos órgãos de algumas espécies de plantas para sinalizar padrões de diversidade morfofisiológica e a magnitude das variações fenotípicas das plantas entre diferentes ambientes de um mesmo bioma (Melo Júnior 2015, Melo Júnior \& Boeger 2015, 2016). Nos ambientes de Restinga, os resultados apontaram que as mais evidentes variações fenotípicas são as morfoanatômicas (massa foliar por área, espessura foliar, densidade foliar, suculência foliar e densidade da madeira), as fisiológicas (concentração de clorofilas a $+\mathrm{b}$, potencial de água na folha, módulo de volume de elasticidade, pressão osmótica e isótopos estáveis de ${ }^{15} \mathrm{~N},{ }^{16} \mathrm{O}$ e ${ }^{1} \mathrm{H}$ ) e as fenológicas (taxas de rotatividade, longevidade e mortalidade de foliar) (Franco et al. 1996, Scarano et al. 2001, Berg et al. 2004, Dias et al. 2006, Cavalin \& de Mattos 2007, Gessler et al. 2008, Rosado \& de Mattos 2010, Rosado et al. 2013 a, b). Já foi descrita, por exemplo, a formação de morfotipos (ou ecótipos) em ambientes de Restinga para Varronia curassavica Jacq. (Boraginaceae), Dodonaea viscosa Jacq. (Sapindaceae) e Symphyopappus casarettoi B.L.Rob. (Asteraceae) (Melo Júnior \& Boeger 2016).

Andira fraxinifolia Benth (Fabaceae) é uma planta lenhosa, perenifólia, heliófila, seletiva higrófita, endêmica no Brasil e característica da Mata Atlântica (Lorenzi 1992). Ocorre em abundância nas florestas tropicais da costa atlântica do país, incluindo as florestas de Restinga (Pennington 1996). Pode ser encontrada desde o Ceará até Santa Catarina, Maranhão e Minas Gerais (Backes \& Irgang 2004). Entretanto, essa espécie pode ocorrer, com menor frequência, em florestas latifoliadas no planalto, em vegetações secundárias e no interior de florestas primárias densas (Lorenzi 1992). No sul do país, $A$. fraxinifolia pode ser encontrada em fitofisionomias litorâneas de floresta atlântica, destacando-se as restingas. Devido à sua boa propagação e rápida germinação, tem grande importância na recuperação de áreas degradadas (Backes \& Irgang 2004).

Esse estudo analisou as variações em características morfo-anatômicas de Andira fraxinifolia em duas distintas fitofisionomias de Restinga: a Restinga arbustivo-arbórea e a floresta de transição. Esperava-se que a heterogeneidade ambiental existente entre estes ambientes fosse suficientemente intensa para promover a formação de ecótipos nesta espécie.

\section{Material e métodos}

O estudo foi realizado no Parque Estadual do Acaraí, situado no município de São Francisco do Sul/ $\mathrm{SC}\left(26^{\circ} 14^{\prime} 36^{\prime \prime} \mathrm{S}\right.$ e $\left.48^{\circ} 38^{\prime} 17^{\prime \prime} \mathrm{O}\right)$ (figura 1). Esta unidade de conservação tem uma área de aproximadamente 6.667 ha, situada em região fitoecológica de Floresta Ombrófila Densa, que apresenta formações submontana, aluvial, além de manguezais e restingas. As restingas estão ali representadas com quatro diferentes formações fitofisionômicas: Restinga herbácea, Restinga arbustiva, Restinga arbustivoarbórea e floresta de transição (Melo Jr. \& Boeger 2015). O município apresenta clima subtropical úmido, com verões chuvosos e invernos secos (Cfa de Köppen). A temperatura média anual é de $21,3{ }^{\circ} \mathrm{C}$ e o índice pluviométrico varia em torno de $2.273 \mathrm{~mm}$ anualmente (Climate-data 2016).

As coletas de material vegetal e dados ambientais foram realizadas no mesmo período do ano, entre os meses de janeiro e fevereiro de 2014. Os espécimes de Andira fraxinifolia foram coletados nas formações de Restinga arbustiva-arbórea e floresta de transição em parcelas amostrais do módulo RAPELD (PPBio) instalado no parque. A formação arbustiva-arbórea de Restinga é composta por arbustos e árvores entre $2 \mathrm{e}$ $5 \mathrm{~m}$ de altura e apresenta solo do tipo Espodossolo Ferrihumilúvico não hidromórfico. A floresta de transição tem dossel contínuo entre 15 e $20 \mathrm{~m}$ de altura, desenvolve-se sobre solo do tipo Espodossolo Ferrihumilúvico + Organossolo Háplico de natureza hidromórfica e faz limite com as áreas de várzea e de floresta de terras baixas (Melo Jr. \& Boeger 2016), assim tratadas por apresentarem solos arenosos holecênicos (Brietz 2005, Souza \& Luna 2008).

Em cada fisionomia foram selecionados cinco indivíduos, dos quais foram coletadas 25 folhas completamente expandidas e desprovidas de lesões, localizadas no $3^{\circ}$ ou $6^{\circ}$ nós a partir do ápice do ramo e madeira do caule à altura do peito. A altura da planta e o diâmetro à altura do peito foram registrados ainda em campo para cada espécime amostral. As folhas coletadas foram prensadas e secas em estufa, a $70{ }^{\circ} \mathrm{C}$, para determinação da massa seca $(\mathrm{g})$ em balança analítica. Utilizando um paquímetro digital, foram obtidos o comprimento e largura do limbo $(\mathrm{mm})$. A área foliar $\left(\mathrm{cm}^{2}\right)$ foi obtida por meio de imagem digitalizada em scanner de mesa acoplado ao software Sigma Scan Pro 5.0. A área específica foliar (AEF) foi obtida pela razão entre área foliar e massa seca (Witkowski \& Lamont 1991). Na madeira, o 
comprimento e o diâmetro tangencial dos elementos de vasos $(\mu \mathrm{m})$ e a freqüência dos vasos por $\mathrm{mm}^{2}$ foram mensurados em amostras de $n=30$ (Iawa 1989).

Cinco amostras de $15 \mathrm{~cm}$ superficiais dos solos em cada fitofisionomia foram coletadas para avaliação da condição nutricional edáfica, conforme metodologia recomendada pela Embrapa (2013). As análises dos níveis de $\mathrm{pH}$, macronutrientes $(\mathrm{P}, \mathrm{K}, \mathrm{Ca}, \mathrm{Mg}), \mathrm{H}+\mathrm{Al}$ $\left(\mathrm{mmolc} / \mathrm{dm}^{3}\right)$ e $\mathrm{S}$ foram realizadas pelo Laboratório de Análise do Solo e Planta, do Instituto Agronômico de Campinas. A umidade gravimétrica dos solos foi obtida de quinze amostras por fitofisionomia e calculada segundo Embrapa (2013). Todas as amostras
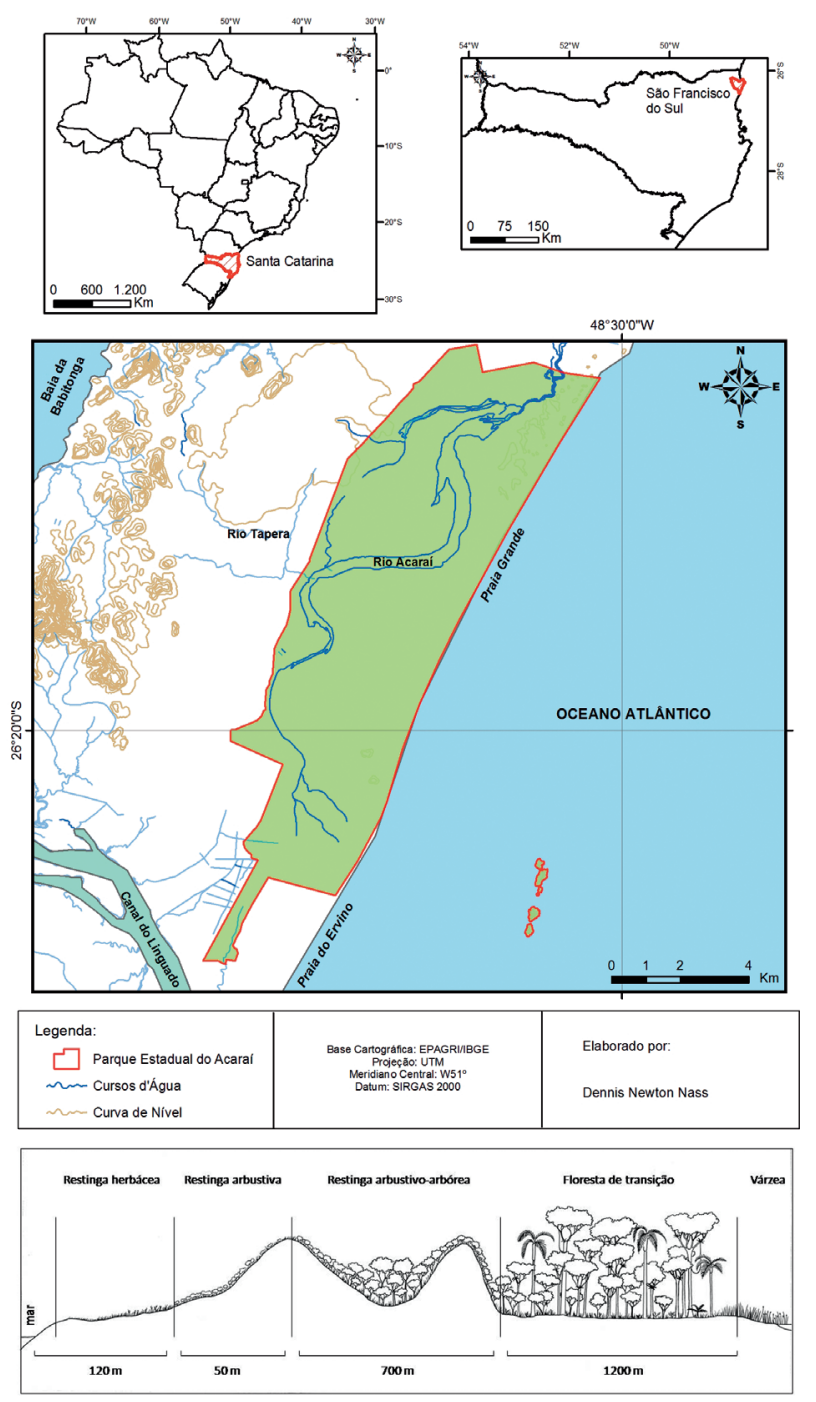

Figura 1. A. Localização da restinga do Parque Estadual do Acaraí, São Francisco do Sul/SC. B: Fitofisionomias de restinga na área estudada. Fonte: Melo Júnior \& Boeger, 2015.

Figure 1. A. Restinga location at Acaraí State Park, São Francisco do Sul/SC . B: Restinga phytophysionomies in the study area. Source: Melo Júnior \& Boeger, 2015. de solo foram coletadas próximas aos indivíduos amostrais. A intensidade luminosa foi estimada com um Light meter, Li-250A (LICOR, USA). Dez medidas foram realizadas próximas às folhas dos indivíduos selecionados, durante a coleta e no mesmo dia.

As amostras vegetais foram processadas no Laboratório de Anatomia e Ecologia Vegetal da Universidade da Região de Joinville (UNIVILLE), de acordo com as técnicas usuais em morfo-anatomia vegetal (Kraus \& Arduim 1997). Amostras de madeira foram cortadas em micrótomo de deslize, clarificadas em hipoclorito de sódio, coradas em safranina etanólica $50 \%$, desidratadas em série alcoólica crescente, fixadas em acetato de butila e montadas sobre resina sintética (Johansen 1940, Sass 1951, Paiva et al. 2006).

O Teste $t$ de Student foi utilizado para comparar os valores médios dos atributos quantitativos entre duas populações estudadas, com o auxílio do software RStudio (Zar 1999, Crawley 2007). A dependência das variáveis foliares e do lenho com as dimensões das árvores foi avaliada pelo teste de correlação de Pearson no mesmo ambiente estatístico. Um índice de variabilidade fenotípica (IVF) foi calculado para todos os atributos foliares e do lenho estudados, segundo a fórmula $\mathrm{IVF}=\left(\mathrm{M}_{\max }-\mathrm{M}_{\min }\right) / \mathrm{M}_{\max }$, onde: $\mathrm{M}_{\max }=$ maior média do atributo e $\mathrm{M}_{\min }=$ menor média do atributo, seguindo as recomendações de Valladares et al. (2006).

\section{Resultados e Discussão}

Os resultados das análises de solo indicaram que ambas as fitofisionomias apresentam solos distróficos, com acidez elevada (tabela 1) em função das somas de bases $(\mathrm{SB}<50)$. O solo da floresta apresentou maiores concentrações de matéria orgânica, assim como uma maior capacidade de troca de cátions quando comparado com o da Restinga arbustivoarbórea. Os teores elevados de alumínio e os baixos valores de $\mathrm{pH}$ em solos ricos em matéria orgânica têm menor impacto negativo no desenvolvimento das plantas, diferente de solos minerais, onde a presença desses elementos podem causar a infertilidade do solo (Valladares et al. 2007). A capacidade de troca de cátions está relacionada à fertilização, já que indica a capacidade total de retenção de cátions, os quais, em geral, estarão disponíveis às plantas (Chaves et al. 2004). Sendo assim, infere-se que o solo da Restinga arbustivo-arbórea seja menos favorável ao desenvolvimento de plantas, pois apresenta menor 
quantidade de nutrientes, quando comparado ao solo da floresta de transição.

Ambos os solos são pobres em cátions básicos solúveis, como $\mathrm{K}, \mathrm{Mg}$ e $\mathrm{Ca}$, sendo que o solo da floresta de transição apresentou maior disponibilidade desses elementos, assim como a de $\mathrm{P}\left(25,67 \mathrm{~mm} . \mathrm{dm}^{-3}\right)$, quando comparado ao da Restinga arbustivo-arbórea $\left(1 \mathrm{~mm} \cdot \mathrm{dm}^{-3}\right.$ ) (tabela 1). A baixa disponibilidade desse elemento pode ser um fator limitante para as plantas, uma vez que desempenha um papel chave nos processos do metabolismo energético (Epstein 1975, Silva \& Delatorre 2009).

Os solos de Restinga caracterizam-se por serem arenosos e pobres em nutrientes, sendo o spray marinho a principal fonte nutritiva (Araújo \& Lacerda 1987, Leão \& Dominguez 2000). Entretanto, apesar do solo de Restinga arbustivo-arbórea ser pobre em nutrientes, muitas plantas parecem ter alta eficiência para a utilização dos minerais (Melo Júnior 2015). Por outro lado, o solo florestal, ainda que muito ácido, tem um alto teor de matéria orgânica, sendo retroalimentado pela substancial produção de serapilheira (Silva et al. 2008). A intensidade luminosa foi equivalente a 841,7 $( \pm 26,6)$ umol.s ${ }^{-1} \cdot \mathrm{m}^{-2}$ na Restinga arbustivo-arbórea e a1451,8 $( \pm 21,7)$ umol.s $\mathrm{s}^{-1} \cdot \mathrm{m}^{-2}$ na floresta de transição. A maior intensidade luminosa da Restinga arbustivoarbórea pode ter sido decorrente do fato de a coleta de dados ter sido realizada em regiões protegidas pela vegetação de dossel, enquanto que na floresta de transição os pontos para coleta de dados encontravamse próximos na região de borda que recebem mais luz.

Considerando os atributos foliares, os valores médios das folhas para massa seca, área, largura e comprimento do limbo foram maiores nas plantas da Restinga arbustivo-arbórea em comparação com os valores médios das folhas de plantas da floresta de transição (tabela 2). Lâminas foliares com maior superfície captam mais energia luminosa, mas necessitam investir mais em tecido de sustentação, o que pode contribuir para o aumento da sua massa (Evert 2013). Esse fato é observado no incremento da massa foliar (36\%) que representou o dobro do incremento em área (18\%), quando os valores médios das folhas das duas formações são comparados. Um maior ajuste na lâmina foi observado no comprimento (74\% de variação) do que na largura foliar $(10 \%$ de variação), o que pode representar um maior investimento no tecido mecânico (Niklas 1996).

Esses ajustes na morfometria da folha se refletiram nos valores médios da área específica foliar (AEF), que foram similares entre as formações (tabela 2). Esse resultado não era esperado, uma vez que a $\mathrm{AEF}$ também responde às condições diferenciadas de luz (Pérez-Harguindeguy et al. 2013). No entanto,

Tabela 1. Caracterização nutricional, espessura da serapilheira e umidade gravimétrica dos solos das formações de Restinga arbustiva-arbórea (Raa) e de Floresta de transição (Ft) do Parque Estadual do Acaraí, São Francisco do Sul/SC. Letras diferentes na mesma linha mostram diferença significativa $(\mathrm{p}<0,05)$.

Table 1. Nutritional characterization, litter thickness, and soil gravimetric moisture from shrub-tree Restinga (Raa) and Forest transition (Ft) at Acaraí State Park, São Francisco do Sul/SC. Different letters in the same roll showed significant differences $(\mathrm{p}<0.05)$.

\begin{tabular}{|c|c|c|}
\hline \multicolumn{3}{|c|}{ Formações } \\
\hline Atributos do solo & Raa & $\mathrm{Ft}$ \\
\hline $\mathrm{MO}\left(\mathrm{g} \cdot \mathrm{dm}^{-3}\right)$ & $14,67^{\mathrm{b}}$ & $82,67^{\mathrm{a}}$ \\
\hline $\mathrm{pH}$ & $3,53^{\mathrm{a}}$ & $3,10^{\mathrm{a}}$ \\
\hline $\mathrm{P}\left(\mathrm{mg} \cdot \mathrm{dm}^{-3}\right)$ & $1,00^{\mathrm{b}}$ & $25,67^{\mathrm{a}}$ \\
\hline $\mathrm{K}\left(\right.$ mmolc. $\left.\mathrm{dm}^{-3}\right)$ & $1,30^{\mathrm{b}}$ & $2,27^{\mathrm{a}}$ \\
\hline $\mathrm{Ca}\left(\mathrm{mmolc} \cdot \mathrm{dm}^{-3}\right)$ & $5,67^{\mathrm{b}}$ & $7,67^{\mathrm{a}}$ \\
\hline $\operatorname{Mg}\left(\right.$ mmolc.dm $\left.{ }^{-3}\right)$ & $1,00^{\mathrm{b}}$ & $2,00^{\mathrm{a}}$ \\
\hline $\mathrm{H}+\mathrm{Al}\left(\right.$ mmolc. $\left.\mathrm{dm}^{-3}\right)$ & $21,67^{b}$ & $291,67^{\mathrm{a}}$ \\
\hline SB $\left(\right.$ mmolc.dm $\left.{ }^{-3}\right)$ & $8,80^{\mathrm{b}}$ & $13,83^{\mathrm{a}}$ \\
\hline CTC (mmolc. $\left.\mathrm{dm}^{-3}\right)$ & $30,47^{b}$ & $305,50^{\mathrm{a}}$ \\
\hline $\mathrm{V}(\%)$ & $29,00^{\text {a }}$ & $4,33^{\mathrm{b}}$ \\
\hline Salinidade (mmolc. $\left.\mathrm{dm}^{-3}\right)$ & $0,83^{\mathrm{b}}$ & $1,90^{\mathrm{a}}$ \\
\hline Serapilheira $(\mathrm{cm})$ & $3,7^{\mathrm{b}}$ & $7,9^{\mathrm{a}}$ \\
\hline Umidade gravimétrica (\%) & $14,1^{\mathrm{b}}$ & $76,2^{\mathrm{a}}$ \\
\hline
\end{tabular}


considerando-se que a AEF é uma relação entre área e massa e que esses atributos apresentaram variações diferenciadas entre as formações, pode-se inferir que existiu uma forte influência do comprimento da lâmina nos valores de AEF. Outro atributo importante que pode atuar sobre os valores de AEF é a espessura do limbo, mas essa também não variou entre as folhas das duas formações (tabela 2).

Embora as diferenças nas médias dos atributos mensurados de xilema entre os indivíduos das duas populações não tenham sido significativas, foram observadas certas características anatômicas do xilema secundário esperadas para a família Fabaceae (Metcalfe \& Chalk 1983, Baas et al. 2000), tais como a presença de elementos de vasos de maior calibre, o que favorece um maior fluxo de água (Carlquist 2001). Concomitantemente, é possível visualizar que os vasos apresentaram baixa freqüência (tabela 2), como é esperado para espécies de florestas tropicais, que apresentam características como menor frequência de vasos e elemento de vasos mais largos e mais longos (Metcalfe \& Chalk 1983, Carlquist 1977, 2001).

Apesar de tender a ser menos plástico, já foram observadas variações na estrutura do xilema secundário, em decorrência da influência ambiental (Baas et al. 2004). Características como diâmetro e comprimento dos elementos de vasos, frequência de vasos, comprimento e espessura da parede das fibras (Baas et al.1983, Carlquist 1977), podem ser influenciadas por fatores ambientais como a disponibilidade hídrica, condições edáficas e intensidade luminosa (Costa et al. 2009).

Os indivíduos estudados diferiram entre as duas formações de Restinga em sua arquitetura, considerando os atributos de altura e diâmetro do caule (tabela 2). Os ajustes das características fisiológicas e anatômicas em plantas podem afetar a produção e alocação de biomassa e, conseqüentemente, o seu crescimento (Maule et al. 1995, Lambers et al. 2008, Poorter et al. 2011). Assim, a capacidade de aclimatação de uma espécie pode ser analisada por meio do seu crescimento, observando-se a altura, o diâmetro do caule e a massa seca dos órgãos vegetativos (Soares 2012). Não foram obtidas correlações significativas entre as variáveis foliares e as do lenho com os dados de altura e diâmetro do caule, visto que todas as combinações apresentaram valores de $r$ iguais ou inferiores a 0,20 ou negativos, exceto a correlação entre a área foliar específica e a altura da planta $(\mathrm{r}=0,55, \mathrm{p}<0,0001)$, para ambas as áreas (tabela 3).

A correlação significativa entre a AEF e a altura dos indivíduos sugere maior investimento dos indivíduos em produção fotossintética e o conseqüente crescimento em altura no ambiente florestal, uma vez que nessa formação há maior disponibilidade

Tabela 2. Valores médios e respectivos desvios-padrão (entre parênteses) das variáveis morfo-anatômicas de Andira fraxinifolia (Fabaceae) do Parque Estadual do Acaraí, São Francisco do Sul/SC. Legenda: Restinga arbustivo-arbórea (Raa), Floresta de transição (Ft), valores do teste $t$ de Student (t) e nível de significância (p) da comparação entre as formações de restinga estudadas.

Table 2. Mean values and respective standard deviation(in parentheses) of the morpho-anatomical traits of Andira fraxinifolia (Fabaceae) from Acaraí State Park, São Francisco do Sul SC. Legend: shrub-tree Restinga (Raa), Forest transition (Ft),values of Student's t-test $(t)$ and significance level $(p)$ of the comparison between the studied restinga vegetations.

\begin{tabular}{lcccc}
\hline Variável & \multicolumn{2}{c}{ Formações } & $\mathrm{t}$ & $\mathrm{p}$ \\
\hline & $\mathrm{Raa}$ & $\mathrm{Ft}$ & & \\
\hline Altura da planta $(\mathrm{m})$ & $4,7(0,83)$ & $5,9(1,59)$ & 9,08 & $<0,0001$ \\
Diâmetro do caule a altura do peito $(\mathrm{cm})$ & $28,0(3,67)$ & $36,2(4,71)$ & 18,72 & $<0,0001$ \\
Massa seca foliar $(\mathrm{g})$ & $0,15(0,05)$ & $0,11(0,06)$ & 4,72 & $<0,0001$ \\
Área foliar $\left(\mathrm{cm}^{2}\right)$ & $13,26(4,5)$ & $11,21(3,06)$ & 4,2 & $<0,0001$ \\
Comprimento foliar $(\mathrm{mm})$ & $118,66(522,8)$ & $68,09(12,10)$ & 2,28 & $=0,01$ \\
Largura foliar $(\mathrm{mm})$ & $23,24(4,70)$ & $20,95(2,87)$ & 4,65 & $<0,0001$ \\
Área específica foliar $\left(\mathrm{cm}^{2} \cdot \mathrm{g}^{-1}\right)$ & $91,52(23,77)$ & $115,04(98,35)$ & 2,59 & $=0,99$ \\
Espessura do limbo $\left(\mathrm{mm}^{-1}\right)$ & $0,23(0,048)$ & $0,24(0,069)$ & 0,42 & $=0,67$ \\
Diâmetro dos vasos $(\mu \mathrm{m})$ & $147,83(35,70)$ & $160,00(38,43)$ & 2,84 & $=0,99$ \\
Comprimento dos elementos de vasos $(\mu \mathrm{m})$ & $261,5(43,73)$ & $258,83(37,45)$ & 0,57 & $=0,28$ \\
Frequência dos vasos $\left(\mathrm{mm}^{2}\right)$ & $2,62(1,02)$ & $2,73(1,22)$ & 0,89 & $=0,81$ \\
\hline
\end{tabular}


de recursos (Vendramini et al. 2002). Na formação de Restinga, onde há maior limitação de recursos nutricionais e água disponível, parece haver maior desenvolvimento de tecidos de sustentação mecânica (Turner 1994), como observado na maior quantidade de massa seca foliar produzida neste ambiente.

Em síntese, pode-se afirmar que as populações de $A$. fraxinifolia diferiram entre as fitofisionomias estudadas quanto ao tamanho das plantas e certas características foliares (tabelas 2 e 4). A população do

Tabela 3. Teste de correlação de Pearson entre as variáveis morfo-anatômicas e as dimensões dos indivíduos de Andira fraxinifolia (Fabaceae) do Parque Estadual do Acaraí, São Francisco do Sul/SC. Legenda: H: altura da planta (m), DAP: diâmetro do caule a altura do peito (cm), AEF: área específica foliar $\left(\mathrm{cm}^{2} \cdot \mathrm{g}^{-1}\right), \mathrm{AF}$ : área foliar $\left(\mathrm{cm}^{2}\right), \mathrm{CF}$ : comprimento foliar (mm), LF: largura foliar (mm), EL: espessura do limbo (mm), MF: massa seca foliar (g), DV: diâmetro tangencial do vaso $(\mu \mathrm{m}), \mathrm{CV}$ : comprimento do elemento de vaso $(\mu \mathrm{m}), \mathrm{FV}$ : frequência de vasos $\left(\mathrm{mm}^{2}\right)$.

Table 3. Pearson correlation test between morpho-anatomical traits and dimensions of individuals of Andira fraxinifolia (Fabaceae) from Acaraí State Park, São Francisco do Sul SC. Legend: H: plant height (m), DAP: stem diameter at breast height $(\mathrm{cm})$, AEF: leaf specific area $\left(\mathrm{cm}^{2} . \mathrm{g}^{-1}\right)$, AF: leaf area $\left(\mathrm{cm}^{2}\right)$, CF: leaf length $(\mathrm{mm})$, LF: leaf width $(\mathrm{mm})$, EL: blade thickness $(\mathrm{mm})$, MF: dry mass $(\mathrm{g}), \mathrm{DV}$ : tangential vessel diameter $(\mu \mathrm{m}), \mathrm{CV}$ : element vessel length $(\mu \mathrm{m}), \mathrm{FV}$ : vessels frequency $\left(\mathrm{mm}^{2}\right)$.

\begin{tabular}{cccccccc}
\hline Variável 1 & Variável 2 & $\mathrm{r}$ & $\mathrm{P}$ & Variável 1 & Variável 2 & $\mathrm{r}$ & $\mathrm{P}$ \\
\hline & AEF & 0,55 & $>0,0001$ & & AEF & 0,03 & 0,54 \\
& AF & 0,08 & $>0,0001$ & AF & $-0,37$ & 0,0001 \\
& CF & 0,16 & $>=0,01$ & CF & $-0,27$ & 0,0001 \\
& EL & 0,05 & 0,85 & & EL & 0,10 & 0,63 \\
& HF & 0,001 & 0,98 & DAP & LF & $-0,44$ & 0,0001 \\
& MF & 0,14 & 0,02 & & MF & $-0,21$ & 0,005 \\
& CV & 0,001 & 0,99 & & CV & 0,15 & 0,005 \\
& DV & 0,03 & 0,60 & & DV & $-0,07$ & 0,18 \\
& FV & 0,13 & 0,02 & FV & 0,09 & 0,09 \\
\hline
\end{tabular}

Tabela 4. Índice de variação fenotípica (IVF) das variáveis morfo-anatômicas de Andira fraxinifolia (Fabaceae) ocorrente nas formações de Restinga arbustivo-arbórea e Floresta de transição do Parque Estadual do Acaraí, São Francisco do Sul/SC.

Table 4. Phenotypic Variation Index (PVI) of the morpho-anatomical traits of Andira fraxinifolia (Fabaceae) from two distinct restinga formations: shrub-tree Restinga and Forest transition from Acaraí State Park, São Francisco do Sul/SC.

\begin{tabular}{lc}
\hline Variáveis morfológicas & IVF \\
\hline Comprimento foliar $(\mathrm{mm})$ & 0,42 \\
Massa seca foliar $(\mathrm{g})$ & 0,26 \\
Diâmetro do caule a altura do peito $(\mathrm{cm})$ & 0,23 \\
Altura da planta $(\mathrm{m})$ & 0,20 \\
Área específica foliar $\left(\mathrm{mm}^{2} \cdot \mathrm{g}^{-1}\right)$ & 0,20 \\
Área foliar $\left(\mathrm{mm}^{2}\right)$ & 0,15 \\
Largura foliar $(\mathrm{mm})$ & 0,09 \\
Diâmetro dos elementos de vasos $(\mu \mathrm{m})$ & 0,07 \\
Espessura do limbo $(\mathrm{mm})$ & 0,04 \\
Frequência dos vasos $(\mu \mathrm{m})$ & 0,04 \\
Comprimento dos elementos de vasos $(\mu \mathrm{m})$ & 0,01 \\
\hline
\end{tabular}


ambiente florestal apresentou maior porte, enquanto a população do ambiente de Restinga arbustivo-arbórea apresentou folhas maiores. Estes pequenos ajustes morfofisiológicos podem representar estratégias de sobrevivência que permitiram a otimização de recursos disponíveis em cada ambiente. Por outro lado, nenhuma diferença morfológica foi detectada nos elementos de vasos (tabela 4), o que sugere que estes apresentem grande herdabilidade ou que as influências do meio não tenham sido intensas o suficiente para gerar divergências fenotípicas entre as populações estudadas (Sterns 1989). Sugere-se que outras características morfo-anatômicas de A. fraxinifolia sejam estudadas para que os resultados descritos sejam confirmados e possam ampliar o conhecimento sobre os padrões de diversidade das espécies de Restinga, além de contribuir com a delimitação de atributos funcionais no âmbito dos estudos sobre a diversidade biológica da Mata Atlântica.

\section{Agradecimentos}

Este trabalho teve apoio financeiro do Fundo de Amparo à Pesquisa da UNIVILLE por meio do processo $\mathrm{n}^{\circ}$. 6055 do primeiro coautor. Os autores agradecem ao Programa de Pesquisas em Biodiversidade - Bioma Mata Atlântica - Núcleo Sul/ $\mathrm{SC}$, à equipe gestora do Parque Estadual Acaraí e ao governo do Estado de Santa Catarina, pela concessão da bolsa de pesquisa à autora.

\section{Literatura citada}

Araújo, D.S.D. 1992. Vegetation types of sandy coastal plains of tropical Brazil: a first approximation. In: U. Seeliger (ed.). Coastal plant communities of Latin America, Academic Press, London, pp. 337-347.

Araújo, D.S.D. \& Lacerda, L.D. 1987. A natureza das restingas. Ciência Hoje 6: 42-48.

Baas, P., Werker, E. \& Fahn, A. 1983. Some ecological trends in vessel characters. IAWA Bulletin New Series 4: 141-159.

Baas, P., Wheller, E. \& Chase, M. 2000. Dicotyledonous wood anatomy and the APG system of angiosperm classification. Botanical Journal of the Linnean Society 131: 3-17.

Baas, P., Wheller, E. \& Chase, M. 2004. Evolution of xylem physiology. In: I. Poole \& A. Hemsley (ed.). Evolution of plant physiology. Elsevier Academic Press, London. pp. 273-295.

Backes, P. \& Irgang, B. 2004. Mata atlântica: as árvores e a paisagem. Paisagem do Sul, Porto Alegre.
Berg, A., Orthen, B., de Mattos, E.A., Duarte, H.M. \&, Lüttge, U. 2004. Expression of crassulacicean acid metabolism in Clusia hilariana Schlechtendal in different stages of development in the Field. Trees 18: 553-558.

Carlquist, S. 1977. Ecological factors in wood evolution: a floristic approach. American Journal of Botany 64: 887-896.

Carlquist, S. 2001. Comparative wood anatomy. Systematic, ecological and evolutionary aspects of dicotyledon wood. Springer-Verlag, London.

Cavalin, P.O. \& de Mattos, E.A. 2007. Spatio-temporal variation of photosynthetic pigments in the CAM tree Clusia hilariana Schlechtendal associated with dry spells during the rainy season in southeastern Brazil. Trees 21: 671-675.

Chaves, L.H.G., Tito, G.A., Chaves, I.B., Luna J.G. \& Silva, P.C.M. 2004. Propriedades químicas do solo aluvial da Ilha de Assunção - Cabrobó (Pernambuco). Revista Brasileira de Ciência do Solo 28: 431-437.

Climate-Data. 2016. Clima: São Francisco do Sul. Disponível em http://pt.climate-data.org/location/28141/ (acesso em 6-I-2016).

Costa, C.G., Callado, C.H., Coradin, V.T.R. \& CarmelloGuerreiro, S.M. 2009. Xilema. In: B. Apezzato-daGlória, \& S.M. Carmello-Guerreiro (ed.). Anatomia vegetal. Editora UFV, Viçosa. pp.129-154.

Crawley, M.J. 2007. The R book. John Wiley \& Sons, Chichester.

Dias, A.T.C., de Mattos, E.A., Vieira, S.A., Azeredo, J.V. \& Scarano, F.R. 2006. Aboveground biomass stock of native woodland on a Brazilian sandy coastal plain: estimates based on the dominant tree species. Forest Ecology and Management 226: 364-367.

EMBRAPA. 2013. Sistema brasileiro de classificação de solos. EMBRAPA, Brasília.

Epstein, E. 1975. Nutrição mineral de plantas: princípios e perspectivas. EDUSP, São Paulo.

Esau, K. 1977. Anatomy of seed plants. John Wiley and Sons, New York.

Evert, R.F. 2013. Anatomia das plantas de Esau: meristemas, células e tecidos do corpo da planta: sua estrutura, função e desenvolvimento. Blucher, São Paulo.

Franco, A.C., Haag-Kerwer, A., Herzog, B., Grams, T.E.E., Ball, E., de Mattos, E.A., Scarano, F.R., Barreto, S., Garcia, M.A., Mantovani, A. \& Lüttge, U. 1996. The effect of light levels on daily patterns of chlorophyll fluorescence and organic acid accumulation in the tropical CAM tree Clusia hilariana. Trees 10: 240-247. 
Gessler, A., Nitschke, R., Mattos, E.A., Zaluar, H.L.T., Scarano, F.R., Rennenberg, H. \& Lüttge, U. 2008. Comparison of the performance of three different ecophysiological life forms in a sandy coastal resting ecosystem of SE-Brazil: a nodulated N2-fixing C3- shurb (Andira legalis (Vell.) Toledo, a CAMshurb (Clusia hilariana Schltdl.) and a tap root C3hemicryptophyte (Allagoptera arenaria (Gomes) O. Ktze.). Trees 22: 105-119.

Gratani, L., Covone, F. \& Larcher, W. 2006. Leaf plasticity in response to light of three evergreen species of the Mediterranean maquis. Trees 20: 549-558.

IAWA Committee. 1989. List of microscopic features for hardwood identification. IAWA Bulletin 10: 219-332.

Johansen, D.A. 1940. Plant microtechnique. McGraw-Hill Book Company Inc., New York.

Kedrov, G.B. 2012. Functioning wood. Wulfenia 19: 57-95.

Kraus, J.E. \& Arduin, M. 1997. Manual básico de métodos em morfologia vegetal. Seropédica, Rio de Janeiro.

Lambers, H., Chapim III, F.S. \& Pons, T.L. 2008. Plant physiological ecology. Springer, Berlin.

Leão, Z.M.A.N. \& Dominguez, J.M.L. 2000. Tropical coast of Brazil. Marine Pollution Bulletin 41: 112-122.

Lorenzi, H. 1992. Árvores brasileiras: manual de identificação e cultivo de plantas arbóreas nativas do Brasil. Editora Plantarum, Nova Odessa.

Maule, H.G., Andrews, M., Morton, J.D., Jones, A.V. \& Daly, G.T. 1995. Sun/shade acclimation and nitrogen nutrition of Tradescantia fluminensis, a problem weed in New Zealand native forest remnants. New Zealand Journal of Ecology 19: 35-46.

Melo Júnior, J.C.F. 2015. Plasticidade fenotípica e diversidade funcional de comunidades florísticas em gradiente edáfico na restinga do Parque Estadual do Acaraí, São Francisco do Sul/SC. Tese de Doutorado. Universidade Federal do Paraná, Curitiba.

Melo Júnior, J.C.F \& Boeger, M.R.T. 2015. Riqueza, estrutura e interações edáficas em um gradiente de restinga do Parque Estadual do Acaraí, Estado de Santa Catarina, Brasil. Hoehnea 42: 207-232.

Melo Júnior, J.C.F \& Boeger, M.R.T. 2016. Leaf traits and plastic potential of plant species in a light-edaphic gradient from a restinga in southern Brazil. Acta Biológica Colombiana 21: 51-62.

Metcalfe, C.R. \& Chalk, L. 1983. Anatomy of the Dicotyledons. v. 2. Clarendon Press, Oxford.

Niklas, K.J. 1996. Differences between Acer saccharum leaves from open and wind-protected sites. New Phytologist 143: 19-31.

Paiva, J.G.A, Fank-de-Carvalho, S.M., Magalhães, M.P., Graciano-Ribeiro, D. 2006. Verniz vitral incolor 500: uma alternativa de meio de montagem economicamente viável. Acta Botanica Brasilica 20: 257-264.
Pennington, R.T. 1996. Molecular and morphological data provide phylogenetic resolution at different hierarchical levels in Andira. Journal Systematic of Biology 45: 496-515.

Peppe, D.J., Royer, D.L., Cariglino, B., Oliver, S.Y., Newman, S., Leight, E., Enikolopov, G., FernandezBurgos, M., Herrera, F., Adams, J.M., Correa, E., Currano, E.D., Erickson, J.M., Hinojosa, L. F., Hoganson, J.W., Iglesias, A., Jaramillo, C.A., Johnson, K.R., Jordan, G.J., Kraft, N.J.B., Lovelock, E.C., Lusk, C.H., Niinemets,U., Peñuelas, J., Rapson, G., Wing, S.L. \& Wright, I.J. 2011. Sensitivity of leaf size and shape to climate: global patterns and paleoclimatic applications. New Phytologist 190: 724-739.

Pérez-Harguindeguy, N., Díaz, S., Garnier, E., Lavorel, S., Poorter, H., Jaureguiberry, P., Bret-Harte, M.S., Cornwell, W.K., Craine, J.M., Gurvich, D.E., Urcelay, C., Veneklaas, E.J., Reich, P.B., Poorter, L., Wright, I.J., Ray, P., Enrico, L., Pausas, J.G., de Vos, A.C., Buchmann, N., Funes, G., Quétier, F., Hodgson, J.G., Thompson, K., Morgan, H.D., ter Steege, H., van der Heijden, M.G.A., Sack, L., Blonder, B., Poschlod, P., Vaieretti, M.V., Conti, G., Staver, A.C., Aquino, S. \& Cornelissen, J.H.C. 2013. New handbook for standardized measurement of plant functional traits worldwide. Australian Journal of Botany 61: 167-234.

Poorter, H., Niklas, K.J., Reich, P.B., Oleksyn, J., Poot, P. \& Mommer, L. 2011. Biomass allocation to leaves, stems and roots: meta-analyses of interspecific variation and environmental control. New Phytologist 193: 1-21.

Rosado, B.H.P. \& De Mattos, E.A. 2010. Interspecific variation of functional traits in a CAM-tree dominated sandy coastal plain. Journal of Vegetation Science 21:43-54.

Rosado, B.H.P., Dias, A.T.C. \& de Mattos, E.A. 2013 a. Going back to basis: importance of ecophysiology when choosing functional traits for studying communities and ecosystems. Natureza \& Conservação 11: 15-22.

Rosado, B.H.P., de Mattos, E.A. \& Sternberg, L.S.L. 2013b. Area leaf physiological traits related to leaf water isotopic enrichment in restinga Woody species? Anais da Academia Brasileira de Ciências 85: 1035-1046.

Sass, J.E. 1951. Botanical microtechnique. 2 ed. Iowa State College Press, Ames.

Scarano, F.R. 2002. Structure, function and floristic relationships of plant communities in stressful Habitats to the brazilian atlantic rainforest. Annals of Botany 90: 517-524.

Scarano, F.R., Duarte, H.M., Ribeiro, K.T., Rodrigues, P.J.F.P., Barcellos, E.M.B., Franco, A.C., Brulfert, J., Deleâens, E. \& Luèttge, U. 2001. Four sites with contrasting environmental stress in southeastern Brazil: relations of species, life form diversity, and geographical distribution to ecophysiological parameters. Botanical Journal of the Linnean Society 136: 345-364. 
Schlichting, C.D. 1986.The Evolution of phenotypic plasticity in plants. Annual Review Ecology System 17: 667-693.

Silva, A.A. \& Delatorre, C.A. 2009. Root architecture modification in response to phosphorus and nitrogen availability. Revista de Ciências Agroveterinárias 8: 152-163.

Silva, B.A.O., Ferreira, D.M., Macedo, M. \& Andrade, P. 2008. Elementos de ecologia e conservação. Fundação CECIERJ, Rio de Janeiro.

Soares, M.G. 2012. Plasticidade fenotípica de plantas jovens de Handroanthus schrysotrichus (Mart ex DC.) Mattos (Bignoniaceae) em resposta a radiação solar. Dissertação de Mestrado, Universidade Federal do Espírito Santo, Vitória.

Souza, C.R.G \& Luna, G.C. 2008. Unidades quaternárias e vegetação nativa de planície costeira e baixa encosta da Serra do Mar no litoral norte de São Paulo. Revista do Instituto Geológico 29: 1-18.
Stearns, A.D. 1989. The evolutionary significance of henotypic plasticity. BioScience 39: 436-445.

Turner, J.M. 1994. Sclerophylly: primarily protective? Functional Ecology 8: 669-675.

Valladares, F., Sanchez-Gomez, D. \& Zavala, M.A. 2006. Quantitative estimation of phenotypic plasticity: bridging the gap between the evolutionary concept and its ecological applications. Journal of Ecology 94: 1103-1116.

Valladares, F., Gianoli, E. \& Gómez, J. M. 2007. Ecological limits to plant phenotypic plasticity. New Phytologist 176: 749-763.

Vendramini, F., Díaz, S., Gurvich, D.E., Wilson, P.J., Thompson, K. \& Hodgson, J.G. 2002. Leaf traits as indicators of resource-use strategy in floras with succulent species. New Phytologist 154:147-157.

Witkowski, E.T.F. \& Lamont, B.B. 1991. Leaf specific mass confounds leaf density and thickness. Oecologia 88: 486-493.

Zar, J.H. 1999. Biostatistical Analysis. Prentice-Hall, New Jersey. 